\title{
The role of IRAKI in breast cancer patients treated with neoadjuvant chemotherapy
}

This article was published in the following Dove Medical Press journal:

OncoTargets and Therapy

\section{Muwen Yang' \\ Xingsong Qin' \\ Guangyuan Qin² \\ Xinyu Zheng ${ }^{1,2}$}

'Department of Breast Surgery, First Affiliated Hospital, China Medical University, Shenyang, Liaoning, China; ${ }^{2}$ Breast Cancer Institute of the First Affiliated Hospital, China Medical University, Shenyang, Liaoning, China
Correspondence: Xinyu Zheng Department of Breast Surgery, First Affiliated Hospital, China Medical University, I 55 North Nanjing Street, Shenyang, Liaoning I I000I, China Email xyzheng@cmu.edu.cn
Background: IRAK1 has been shown to be abnormally expressed in a set of tumors leading to tumorigenesis and progression. IRAK 1 is a therapeutic target that drives breast cancer metastasis and resistance to paclitaxel. However, the exact role of IRAK1 in neoadjuvant chemotherapy (NCT) for breast cancer remains unclear. The aim of this study was to investigate the relationship between the expression of IRAK1 and the clinicopathological parameters and survival prognosis of breast cancer patients treated with NCT.

Patients and methods: Based on the clinical data and mRNA microarray data from 1,085 breast cancer patients in The Cancer Genome Atlas, the correlation between IRAK1 expression and clinicopathological parameters of breast cancer was analyzed. Immunohistochemistry was performed to evaluate the expression of IRAK1. The Human Protein Atlas and the String database were used to analyze the expression of IRAK1 protein and its interaction with altered neighboring proteins in breast cancer. IRAK1 alteration was analyzed in cBioPortal database. GEO enrichment of IRAK1 was performed using WEB-based Gene SeT AnaLysis Toolkit.

Results: The expression of IRAK1 was significantly downregulated following NCT. The decreased expression of IRAK1 following NCT was positively correlated with reduced tumor size. Finally, survival analysis confirmed that a shorter survival period was correlated to higher expression of IRAK1 both before and after NCT.

Conclusion: These findings advanced our understanding about the expression pattern of IRAK1 in breast cancer, especially in those patients who were treated with NCT, suggesting that IRAK1 could be used as a prognostic indicator, as well as a potential indicator for evaluating NCT efficacy for breast cancer patients.

Keywords: IRAK1, breast cancer, neoadjuvant chemotherapy, bioinformatics, survival

\section{Introduction}

Breast cancer has the highest incidence among Chinese women, accounting for $15 \%$ of new cancers in women. ${ }^{1}$ Breast cancer is the leading cause of death in women younger than 45 years old. ${ }^{1}$ According to different expression status of receptors including estrogen receptor (ER), progesterone receptor (PR), and Her-2, breast cancer can be divided into four molecular subtypes: luminal A type, luminal B type, triple-negative type, and Her-2 overexpression type. Among them, triple-negative breast cancer has the worst prognosis and is still lacking effective prognostic indicators. ${ }^{2}$ Neoadjuvant therapy (NAT) refers to anti-tumor treatment for patients before surgery. For breast cancer, NAT includes neoadjuvant chemotherapy (NCT), neoadjuvant hormonotherapy, and neoadjuvant Her-2 targeted therapy. ${ }^{3}$ NCT has become the standard treatment for patients with locally advanced breast cancer. After NCT, some inoperable breast cancers can be treated with radical surgery. NCT makes breast-conserving surgery possible by 
reducing the size of primary tumors. In patients who initially need axillary lymph node dissections, NCT also increases the possibility of a separate sentinel lymph node biopsy. ${ }^{4-7}$ Because endocrine therapy is ineffective, chemotherapy is extremely important for triple-negative breast cancer. Unfortunately, there is still a lack of effective predictors for NCT.

There are four members in the IRAK family, including IRAK1, IRAK2, IRAKM, and IRAK4. The IRAK1 gene consists of 14 exons and is located on the $\mathrm{X}$ chromosome. ${ }^{8}$ IRAK1 is the first member of this kinase family and is a key component of the IL-1R signaling pathway. In addition, IRAK1 is a downstream effector molecule of the TLR signaling pathway. The abnormal activation of TLR signaling has an important impact on oncogenesis, allergies, septicemia, and autoimmunity. ${ }^{9}$ In recent years, reports on the relationship between IRAK1 and lung cancer, liver cancer, thyroid cancer, melanoma, and lymphoma have been frequently published. ${ }^{10-13}$ By using bioinformatics methods, Pilarsky et al found that IRAK1 is one of the overexpressed genes commonly found in solid tumors. ${ }^{14}$ Wee et al confirmed that IRAK1 is highly expressed in triple-negative breast cancer compared to other types, and inhibition of IRAK1 expression can effectively suppress the growth and metastasis of triple-negative breast cancer. ${ }^{15}$ However, the exact role of IRAK1 in NCT for breast cancer remains unclear. In this study, we aimed to study the expression pattern of IRAK1 protein in breast cancer, and explore the relationship between IRAK1 expression and clinicopathological characteristics in breast cancer patients treated with NCT.

\section{Patients and methods}

\section{Patients and tissue samples}

A total of 75 primary female breast cancer patients who received NCT treatment at First Hospital of China Medical University from April 2007 to February 2011 were selected for the study. According to WHO standards, all cancers were confirmed as invasive breast cancer by histopathology. ${ }^{16}$ Formalin-fixed, paraffin-embedded core biopsy specimens and resection specimens were stored in the Department of Pathology. ER, PR, HER-2, and Ki67 status were routinely examined and could be obtained from the electronic medical records. Male patients, patients with other types of breast cancer or with history of any malignancy were excluded from our study. The clinicopathological parameters of the 75 patients were summarized in Table 1. Follow-up was updated by review of records and telephone calls. The median follow-up was 49 months. The date of death and the date of relapse were used to calculate disease-free survival (DFS). Formalin-fixed, paraffin-embedded breast tissues were collected from core biopsies (pre-NCT) and matched with resection tissues (postNCT). Six patients achieved pathological complete response (pCR). This study was approved by the Institutional Ethics Committees of the First Affiliated Hospital of China Medical University. All patients received an explanation of the study aims and signed written informed consent. The study was conducted in accordance with the Declaration of Helsinki.

The breast cancer mRNA expression dataset was downloaded and processed from the The Cancer Genome

Table I Clinicopathological characteristics of the patients

\begin{tabular}{|c|c|}
\hline & $\mathbf{N}(\%)$ \\
\hline \multicolumn{2}{|l|}{ Age } \\
\hline$<45$ & $42(56)$ \\
\hline$>45$ & $33(44)$ \\
\hline \multicolumn{2}{|l|}{ Pre-NAC stage (based on ultrasound) } \\
\hline T2 & $38(50.7)$ \\
\hline T3 & $21(28)$ \\
\hline T4 & $16(21.3)$ \\
\hline \multicolumn{2}{|l|}{ Post-NAC stage (based on resection pathology) } \\
\hline T0 & $5(6.7)$ \\
\hline $\mathrm{TI}$ & $21(28)$ \\
\hline T2 & $3 I(4 I .3)$ \\
\hline T3 & $10(13.3)$ \\
\hline T4 & $8(10.7)$ \\
\hline \multicolumn{2}{|l|}{ Tumor size change } \\
\hline Increase & $4(5.3)$ \\
\hline Decrease & $66(88)$ \\
\hline $\mathrm{pCR}$ & $5(6.7)$ \\
\hline \multicolumn{2}{|l|}{ Chemotherapy regimens } \\
\hline Platinum + platinum (TP) & $28(37.3)$ \\
\hline Platinum + cyclophosphamide (TC) & $15(20)$ \\
\hline 5-fluorouracil + epirubicin + cyclophosphamide (CEF) & $32(42.7)$ \\
\hline Axillary lymph node metastasis & $52(69.3)$ \\
\hline ER positive & $3 I(4 I .3)$ \\
\hline Her-2 positive & $44(58.7)$ \\
\hline \multicolumn{2}{|l|}{ Surgery } \\
\hline Breast conserving & $3(4)$ \\
\hline Mastectomy & $72(96)$ \\
\hline \multicolumn{2}{|l|}{ Follow-up } \\
\hline Median: 49 & Range $32-130$ \\
\hline Recurrence & $29(38.6)$ \\
\hline Death & $21(28.0)$ \\
\hline
\end{tabular}

Abbreviations: ER, estrogen receptor; NAC, neoadjuvant chemotherapy; $P C R$, pathological complete response. 
Atlas (TCGA) database (https://cancergenome.nih.gov). Online survival analysis was performed using K-M plotter (http://kmplot.com/analysis/). We only included breast cancer patients who had complete RNAseq data and complete clinical information including tumor size, lymph node status, TNM stage, ER, PR, HER-2, and overall survival (OS) data. Finally, 1,085 cases of breast cancer patients were included in our study. The RNAseq data of all 291 normal samples were used for the comparison of mRNA expression difference between tumor and normal tissues. Relevant clinical and pathological parameters were described in Table 2.

\section{Immunohistochemical staining}

Immunohistochemistry was performed with $4 \mu \mathrm{m}$ formalinfixed, paraffin-embedded sections. After deparaffinization and rehydration, $3 \% \mathrm{H}_{2} \mathrm{O}_{2}$ solution was used to block endogenous peroxidase at room temperature. Antigen retrieval was performed by using sodium citrate buffer, and normal serum was applied to block non-specific antibody binding (reagent B; UltraSensitive ${ }^{\mathrm{TM}} \mathrm{SP}$ immunohistochemistry kit; Maxim Biotech Inc., Rockville, MD, USA). Slides were then incubated overnight at $4^{\circ} \mathrm{C}$ with the primary antibody against IRAK1 (F-4, 1:50 dilution) obtained from Santa Cruz Biotechnology Inc. ([sc-5288] Dallas,

Table 2 Correlation between IRAKI expression and clinicopathological parameters based on The Cancer Genome Atlas database

\begin{tabular}{|l|l|l|l|l|}
\hline \multirow{2}{*}{ Characteristics } & \multirow{2}{*}{ N } & \multicolumn{2}{|l|}{ IRAKI } & \multirow{2}{*}{-value } \\
\cline { 3 - 4 } & & Positive & Negative & \\
\hline Molecular type & & & & $<0.00$ I \\
\hline Luminal A & 419 & 58 & 36 I & \\
\hline Luminal B & 180 & 28 & 152 & \\
\hline Her-2 & 62 & 15 & 47 & \\
\hline Basal & 140 & 22 & 118 & \\
\hline Pathology M stage & & & & 0.048 \\
\hline M0 & 869 & 120 & 749 & \\
\hline MI & 21 & 17 & 4 & \\
\hline Pathology N stage & & & & 0.284 \\
\hline N0 & 488 & 44 & 444 & \\
\hline NI & 355 & 59 & 296 & \\
\hline N2 & 112 & 22 & 90 & \\
\hline N3 & 76 & 15 & $6 I$ & \\
\hline Pathology T stage & & & & 0.809 \\
\hline TI & 277 & 33 & 244 & \\
\hline T2 & 603 & 77 & 526 & \\
\hline T3 & 131 & 25 & 106 & \\
\hline T4 & 37 & 15 & 22 & \\
\hline
\end{tabular}

TX, USA). After overnight incubation, samples were then treated with reagent $\mathrm{C}$ and reagent $\mathrm{D}$ (UltraSensitive ${ }^{\mathrm{TM}} \mathrm{SP}$ immunohistochemistry kit; Maxim Biotech Inc.), respectively. Finally, DAB staining was performed and the staining intensity was graded.

The intensities of IRAK1 staining were scored as 0 (no staining), 1 (weak staining), 2 (medium staining), and 4 (strong staining). The results were evaluated and scored independently by at least two pathologists in a blinded manner using a semi-quantitative scoring system considering the extent and intensity. The mean percentage of positive cells was scored as $0(0 \%), 1(1 \%-30 \%), 2(31 \%-50 \%), 3(51 \%-80 \%)$, and 4 (81\%-100\%). The two scores were multiplied, resulting in a final immunoreactivity score ranging between 0 and 12 . The six patients who achieved pCR were regarded as negative postneoadjuvant chemotherapy (NAC).

\section{Statistical analysis}

All the statistical analyses were carried out using SPSS version 23.0 software and Graphpad 6.0. Wilcoxon signedrank test was used to analyze the difference in IRAK1 expression before and after NCT. Mann-Whitney U test was carried out to assess the difference in expression change between group A and group B (divided according to the decrease in tumor size). Spearman's correlation was used to reveal the correlation between the expression level of IRAK1 and the clinicopathological characteristics. ROC curves were drawn to find the cutoff values (giving the highest combined sensitivity and specificity) to dichotomize pre- and post-NAC expression scores of IRAK 1 for the end point of DFS. DFS was estimated using Kaplan-Meier analyses. Additionally, multivariate Cox linear analysis was carried out between the expression of IRAK1 and clinicopathological features, including tumor grade, receptor status, pre-NAC tumor stage, and axillary metastasis. All $P$-values presented are two-sided, and $P \leq 0.05$ was considered to be statistically significant.

\section{Results}

\section{The expression pattern of IRAKI in breast cancer}

We first investigated the difference in mRNA expression for IRAK1 using the mRNA HiSeq expression data from TCGA. As shown in Figure 1A, IRAK1 expression was upregulated in breast cancer tissues compared with the normal breast tissues. The Human Protein Atlas is dedicated to providing information on the organization and cell distribution of all 24,000 human proteins and provides free access to the 


\section{A}

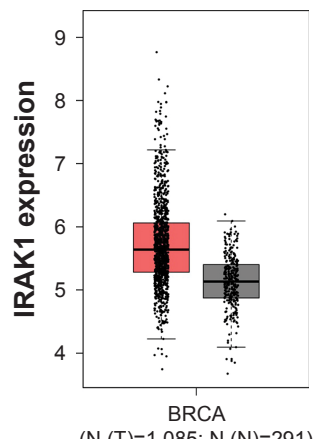

B

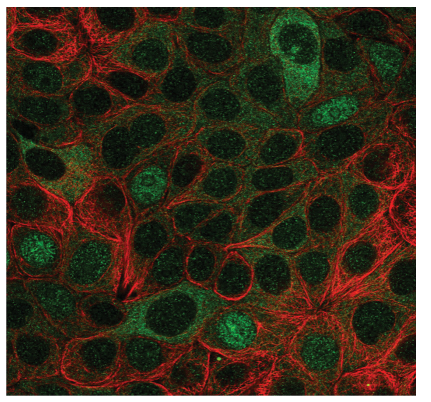

C

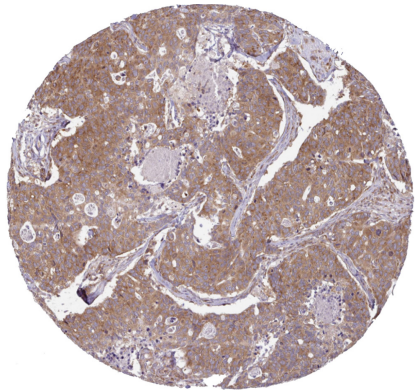

$(\mathrm{N}(\mathrm{T})=1,085 ; \mathrm{N}(\mathrm{N})=291)$

Figure I IRAKI expression in The Cancer Genome Atlas (TCGA) database and The Human Protein Altas.

Notes: (A) IRAKI was highly expressed in breast cancer tissues compared with normal breast tissues based on TCGA database. (B) IRAKI expressed in MCF-7 cell line. (C) IRAKI expressed in breast cancer tissue.

public. The Swedish Knut \& Alice Wallenberg Foundation, which founded the database, uses immunohistochemistry with specific antibodies to examine each protein in 48 human normal tissues, 20 tumor tissues, 47 cell lines, and 12 blood cells. The distribution and expression of the results were expressed in at least 576 immunohistochemical stains and read and indexed by a professional. The examined tissues were from 144 different individuals and 216 tumor tissues, ensuring that the staining results were fully representative. ${ }^{17}$ Data in Figure 1B and C showed IRAK1 expressed in breast cancer cells (MCF-7) and breast cancer tissues, respectively.
To further explore the expression pattern of IRAK1 in patients treated with NCT, immunohistochemistry was carried out in 75 patients. IRAK1 was mainly expressed in the cytoplasm. IRAK1 expression level was reduced obviously following NAC treatment in 58/75 patients (77.3\%). Representative staining images of IRAK1 in matched breast tumor tissues (Figure 2A and C) pre- and (Figure 2B and D) post-NAC chemotherapy were shown in Figure 2, Wilcoxon signed rank test revealed a significant difference in IRAK1 expression between pre-NAC and post-NAC (IRAK1, $P<0.001$ ), Figure $3 A$ and B. Spearman's correlation analysis
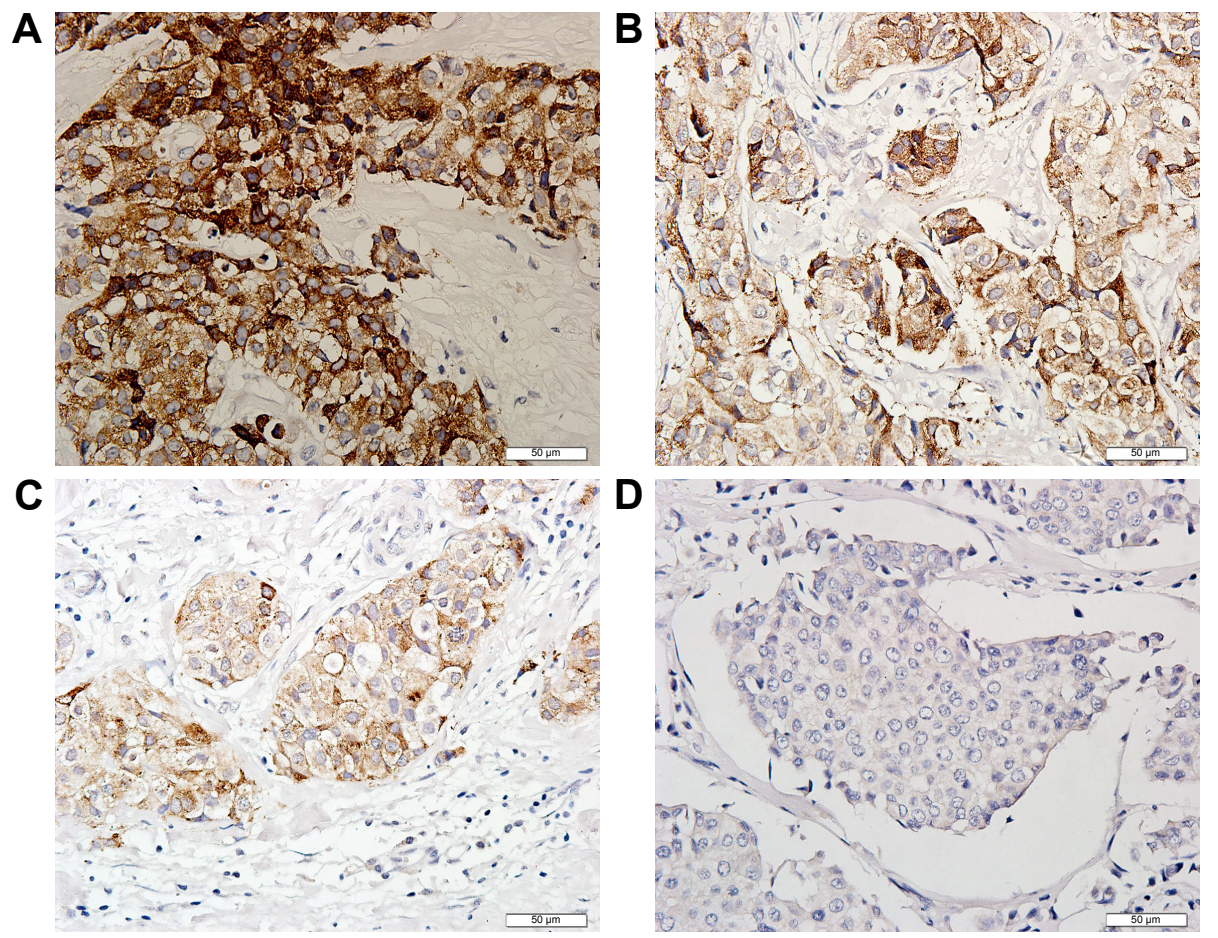

Figure 2 Immunohistochemistry images of IRAKI expression in matched breast tumor tissues pre- and post-neoadjuvant chemotherapy (NCT).

Notes: (A-D) Representative staining images of IRAKI in matched breast tumor tissues pre- and post-NCT. (A) Strongly stained. (B) Moderately stained. (C) Moderately stained. (D) Negatively stained. Magnification $\times 400$. 
A

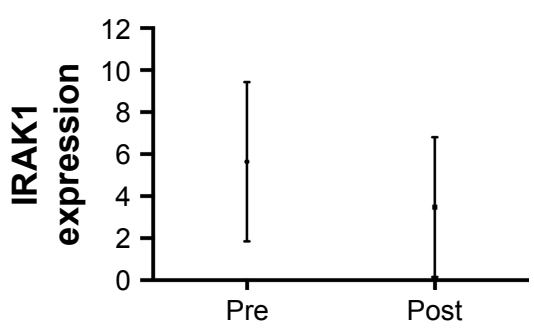

B

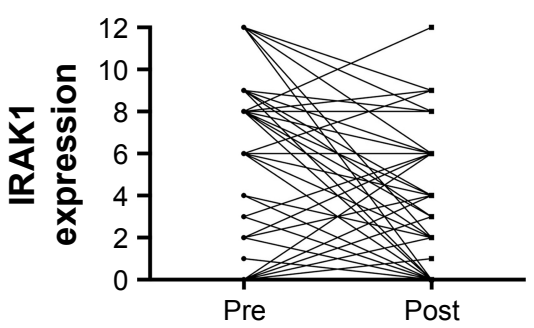

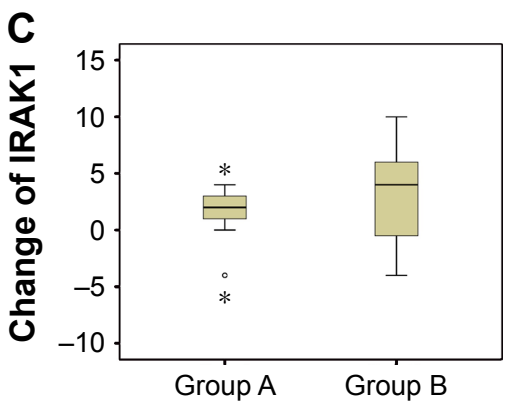

Figure 3 The expression of IRAKI before and after neoadjuvant chemotherapy.

Notes: (A) Difference in expression before and after neoadjuvant chemotherapy. (B) Distribution of the values before and after chemotherapy, respectively. (C) Patients whose tumor sizes diminished by $<2 \mathrm{~cm}$, showed no change or increased, were defined as group $\mathrm{A}$, while patients whose tumor sizes diminished by $>2 \mathrm{~cm}$ or those who achieved pathological complete response were defined as group $B$. The Mann-Whitney $U$ test was used $(P=0.019)$.

was performed between IRAK1 expression (both pre- and post-NAK) and clinicopathological characteristics (including the age when the patient was diagnosed, molecular subtypes, histological grade, TNM stage, ER, PR, HER-2 levels, and lymph node status), and it was shown that the expression of IRAK1 both pre-NAC and post-NAC was not related to the patient's clinicopathological characteristics.

\section{Decrease in IRAKI expression following NAC was associated with reduction of tumor size}

The relationship between the reduction of tumor size and the expression of IRAK1 pre- and post-NAC was investigated. Patients were divided into two groups according to the reduction of tumor size. For patients with tumors that had diminished by $<2 \mathrm{~cm}$, and the patients with an unchanged or increased tumor size were defined as group A, while patients whose tumor sizes had diminished by $>2 \mathrm{~cm}$, or patients who had achieved pCR were defined as another group (group B). Our data showed that there was a significant difference in the decrease of IRAK1 expression between the two groups (Mann-Whitney U test, $P=0.019$, Figure 3C).

\section{The correlation between DFS and the expression of IRAKI pre- and post-NAC treatment}

First, we analyzed the correlation between IRAK1 expression and survival online (www.kmplot.com). In order to assess the differential survival with respect to the expression of IRAK1 pre- and post-NAC, as well as the expression change of IRAK1 following NAC, Kaplan-Meier survival analyses were performed. ROC curve analyses were used to dichotomize the expression scores into high and low expression groups. The cutoff values were obtained from the highest combined sensitivity and specificity at the end point of DFS, and were shown as follows: pre-NAC:7; post-NAC:5. A high expression of IRAK1 both in pre-NAC and post-NAC was found to be significantly correlated with poor DFS. High level of IRAK 1 at post-NAC was more valuable in predicting poor DFS compared with that at pre-NAC (Figure 4).

\section{IRAKI alteration analyzed in cBioPortal database}

The cBio Cancer Genomics Portal (http://cbioportal.org) is an open platform, providing data of more than 5,000 tumor samples from 20 cancer studies, which can help cancer researchers to study in depth. ${ }^{18-20}$ We investigated the genetic alteration of IRAK1 with a cohort of 816 cases of invasive breast cancer available in the cBioPortal database. The result showed that $6.9 \%(56 / 816)$ of cases of breast cancer in this cohort exhibited IRAK1 alteration (Figure 5A). Moreover, a network of the interaction between IRAK1 and several frequently altered neighboring genes was established (Figure 5B). Survival analysis (OS and DFS) was performed to compare the prognosis of the patient groups with and without IRAK1 alteration. However, the results did not show statistical significance (Figure 5C and D).

\section{IRAKI protein expression in the String database}

The String database is a database that is used to search the potential interactions between known proteins and predicted proteins. This database can be applied to 2,031 species and contains interactions between 9.6 million proteins and 13.8 million proteins. ${ }^{21,22}$ An analysis was performed to investigate the correlations between IRAK1 expression and its potential targets. The results revealed that the expressions of several target proteins were significantly correlated with IRAK1 expression according to the GEPIA online database. ${ }^{23}$ (MYD88: $r=0.19$, IKBKG: $r=-0.15$, IL1R1: $r=-0.073$ 


\section{A}

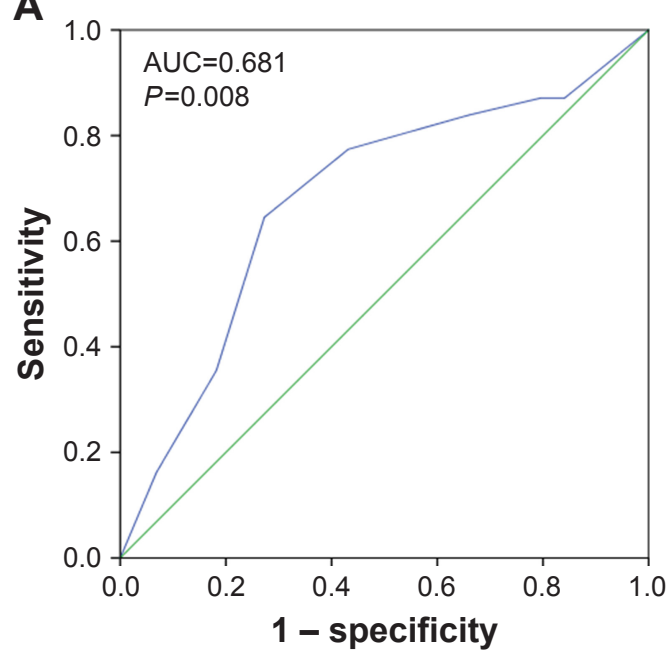

B

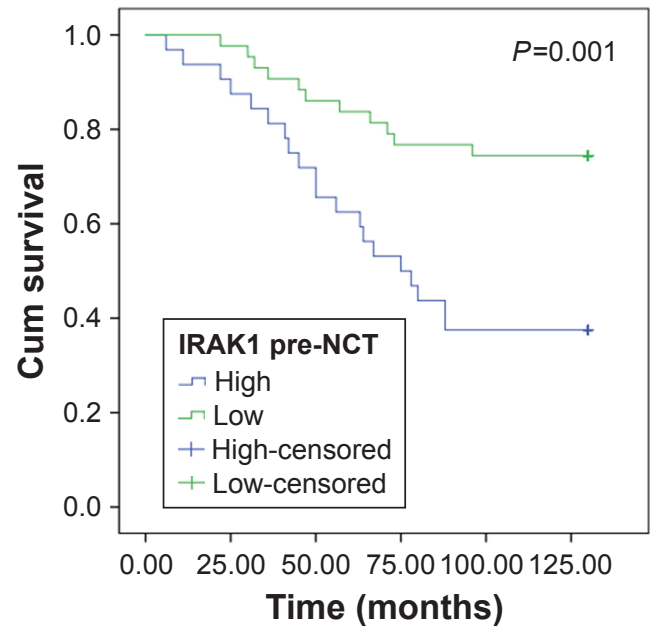

C

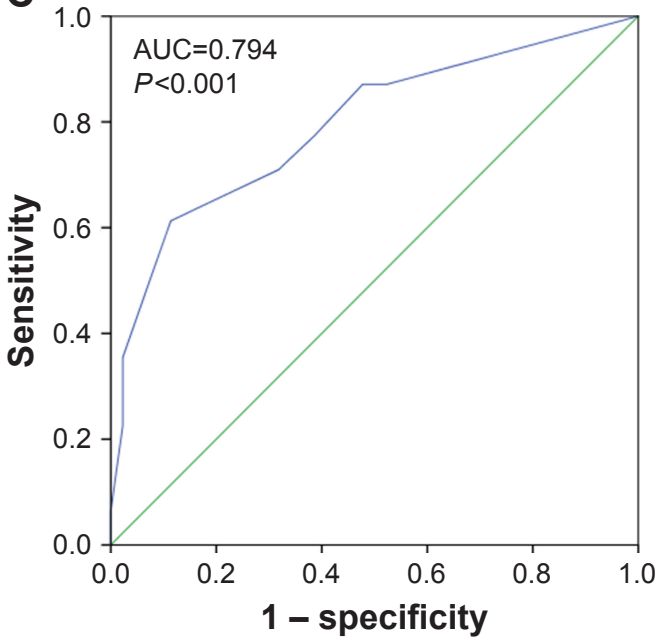

D

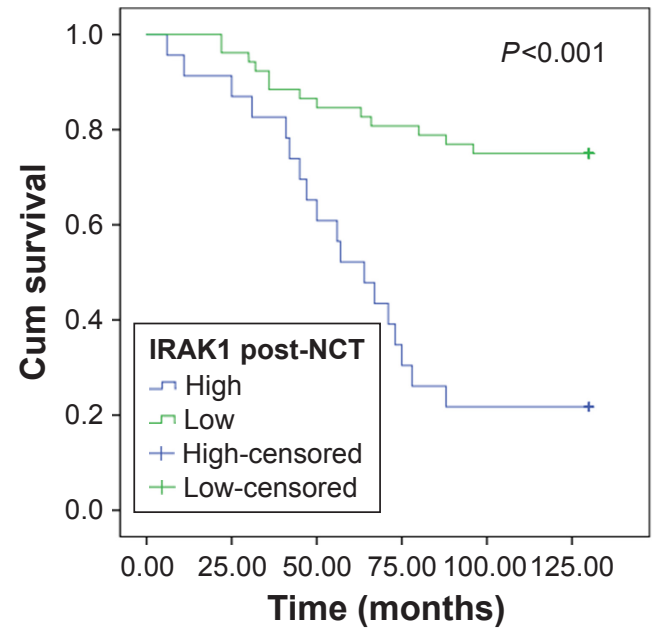

Figure 4 ROC curve and survival curve of IRAKI expression both pre-neoadjuvant chemotherapy (NCT) and post-NCT.

Notes: (A) ROC curve of IRAKI pre-NCT, an AUC value of 0.68 I indicated ( $P=0.008$ ). (B) Kaplan-Meier survival curve of IRAKI pre-NCT (log rank: $P=0.00 \mathrm{I}$ ). (C) ROC curve of IRAKI post-NCT. An AUC value of 0.794 ( $P<0.00$ I). (D) Kaplan-Meier survival curve of IRAKI post-NCT (log rank: $P<0.00 I)$.

TAB1: $r=0.048$, TRAF6: $r=-0.035$, IRAK4: $r=-0.055$, NFKBIA: $r=0.01$, IRF7: $r=0.12$, TAB2: $r=-0.021$, TOLLIP: $r=0.092$, all $P<0.05$ besides TRAF6). Remarkably, MYD88 and IRF7 showed a relatively good correlation with IRAK1 (Figure 6). Both MYD88 and IRF7 play a major role in the TLR4 signaling pathway, which is consistent with Wee et al's study of IRAK1 in breast cancer. This also suggests that IRAK1 may mediate its role in NCT for breast cancer via TLR signaling pathway.

\section{Gene set enrichment analysis of IRAKI in WebGestalt 2017}

WebGestalt (Web-based Gene SeT AnaLysis Toolkit, http://www.webgestalt.org) was one of the first web tools for functional enrichment analysis. ${ }^{24,25}$ The GOSlim classification plot is used to show the distribution of IRAK1 across the major branches of the GO biological process, cellular component and molecular function ontologies. The enrichment results were shown in Figure 7. The results showed that IRAK1 is mainly localized in the cell membrane in breast cancer, mainly enriched in biological regulation, metabolic process, and protein binding, which also paves the way for further research on the mechanism of IRAK1 in NCT for breast cancer in the future.

\section{Discussion}

IRAK1 has been shown to play an important role in the IL-1/TLR signaling pathway. TLRs are key regulators of the innate immune response and have been observed in many malignancies, ${ }^{26}$ including breast cancer. IRAK1, a downstream effector of TLR2, inhibits IRAK1 expression and can also inhibit the growth of breast cancer in vitro and in vivo, indicating that IRAK1 may be a potential therapeutic and prophylactic target for breast cancer through the TLR2 


\section{A}

\section{IRAK1

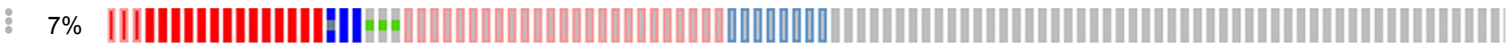
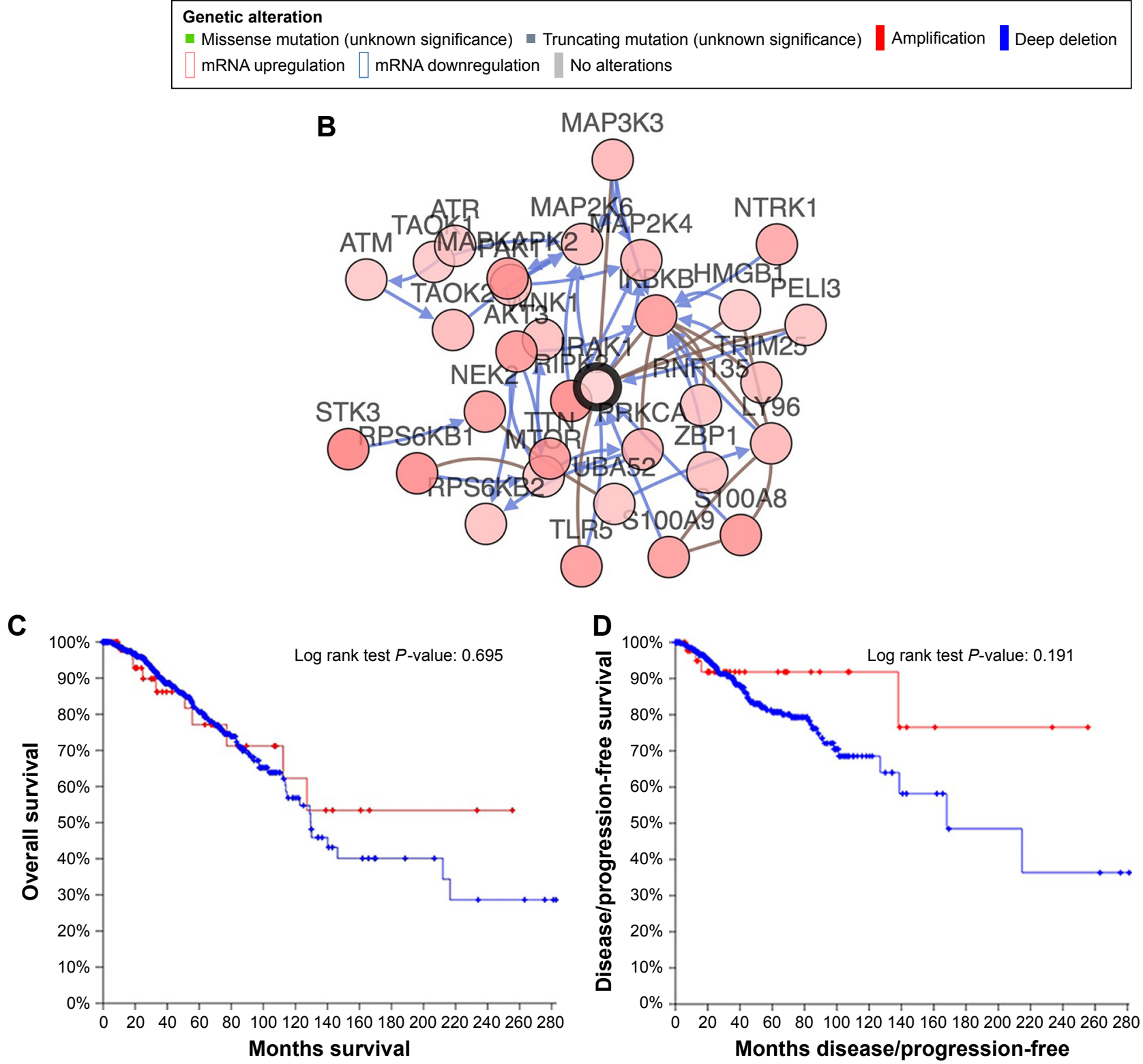

Cases with alteration(s) in query gene(s)

Cases without alteration(s) in query gene(s)

Figure 5 The alteration of IRAKI and its interaction in altered neighboring genes from the cBioPortal database.

Notes: (A) IRAKI gene alteration. In this cohort, 7\% (56/8I6) of invasive breast cancer cases exhibited IRAKI gene alteration. (B) Network of IRAKI and altered neighboring genes. Analysis of the overall survival (C) and disease-free survival (D) of invasive breast cancer patients with and without IRAKI gene alteration. No statistically significant difference was identified.

pathway. ${ }^{27}$ TLR4 overexpression in human breast tumors is often associated with paclitaxel chemoresistance. ${ }^{28}$ In recent years, some studies have confirmed that IRAK1 can act as a proto-oncogene to promote tumor progression, metastasis, and chemotherapy resistance. IRAK1 is overexpressed and over-activated in myelodysplastic syndrome (MDS). Treatment with small-molecule IRAK1 inhibitors (IRAK1/4-Inh) resulted in increased proliferation and apoptosis of clones of
MDS, which was consistent with the inhibition of TRAF6/ NF- $\mathrm{KB}$. These findings suggest that IRAK1 can be used as a therapeutic target for MDS. ${ }^{29}$ The expression level of IRAK1 protein may be used as a parameter to identify whether patients are at high risk for lung cancer and may serve as a potential target for lung cancer diagnosis and gene therapy. ${ }^{13}$ IRAK1 is an oncogene in the development of hepatocellular carcinoma and is overexpressed in 

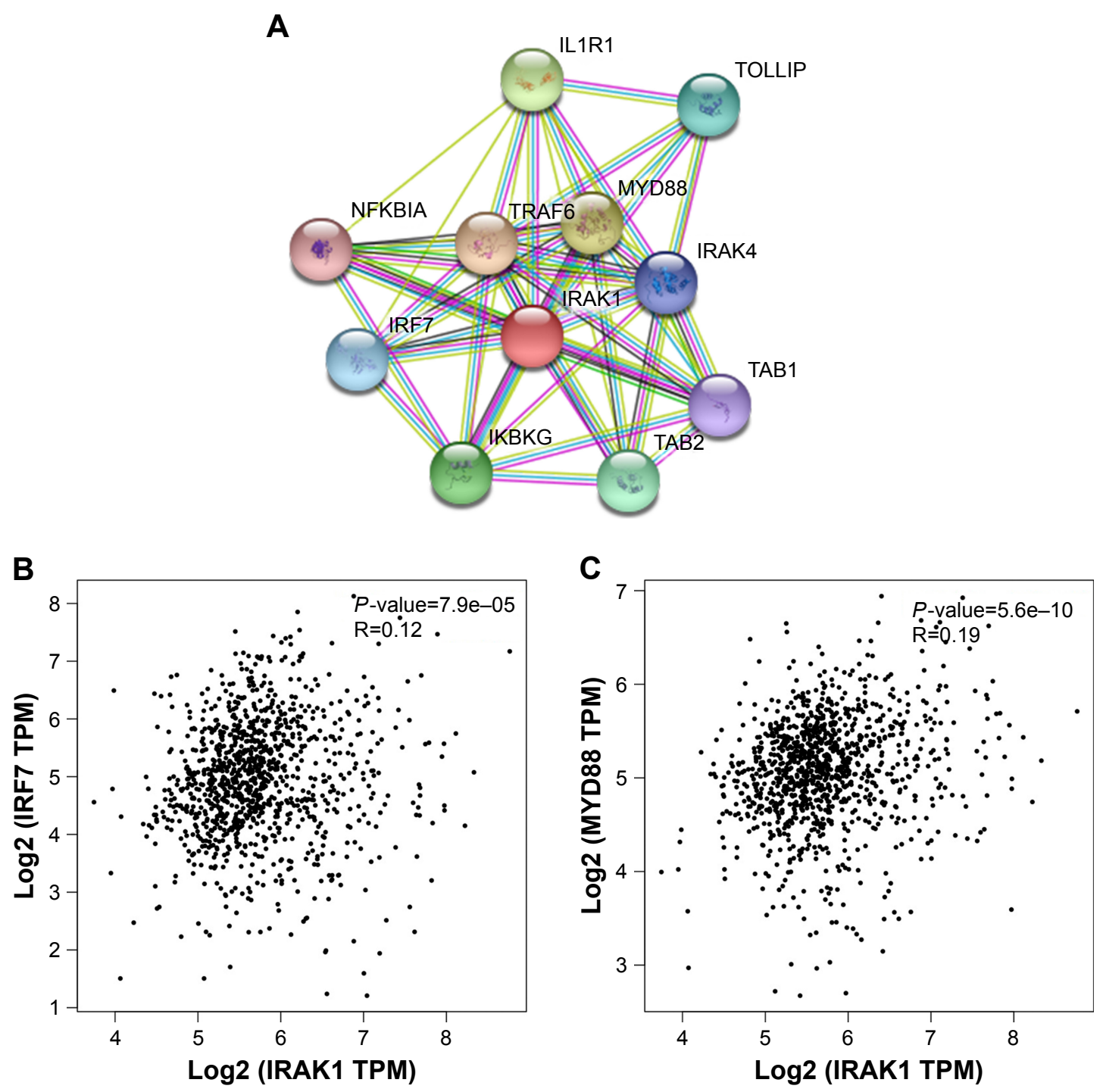

Figure 6 IRAKI protein expression in the String database.

Notes: (A) Corresponding proteins of IRAKI protein in network visualization. (B,

, C) Pearson correlation analysis revealed that IRAKI expression was significantly correlated with the expression of IRF7 and MYD88, respectively.

hepatocellular carcinoma. In addition, by analyzing the immunohistochemistry and RNA sequencing data in cancer genomic maps, IRAK1 is expected to become a diagnostic and therapeutic target for hepatocellular carcinoma. ${ }^{30}$ However, to the best of our knowledge, there has been no study investigating the potential role of IRAK1 in NCT for breast cancer. In this study, we, for the first time, used immunohistochemistry to detect the expression of IRAK1 protein in breast cancer tissues before and after NCT in breast cancer patients undergoing NCT.

Neoadjuvant systemic chemotherapy is a possible method for the treatment of locally advanced, operable, primary non-surgical or inflammatory breast cancer. There is a clear correlation between response to chemotherapy drugs and DFS and OS in breast cancer patients. However, the great challenge for NAC is chemotherapy resistance, and there is also a lack of excellent predictors of NAC response. Although many different prognostic indicators are being developed, few molecular markers are used to predict NAC responses. In this study, we found that changes in IRAK1 expression were correlated with changes in tumor size before and after chemotherapy. Although this finding was not sufficient to predict chemotherapy sensitivity prior to NAC, it can be used as a marker for evaluating the efficacy of NCT and as a reference for postoperative chemotherapy regimens.

In this study, we first studied the correlation between IRAK1 expression and the clinicopathological parameters of breast cancer and its impact on prognosis based on the clinical data and mRNA microarray data of 1,085 breast 

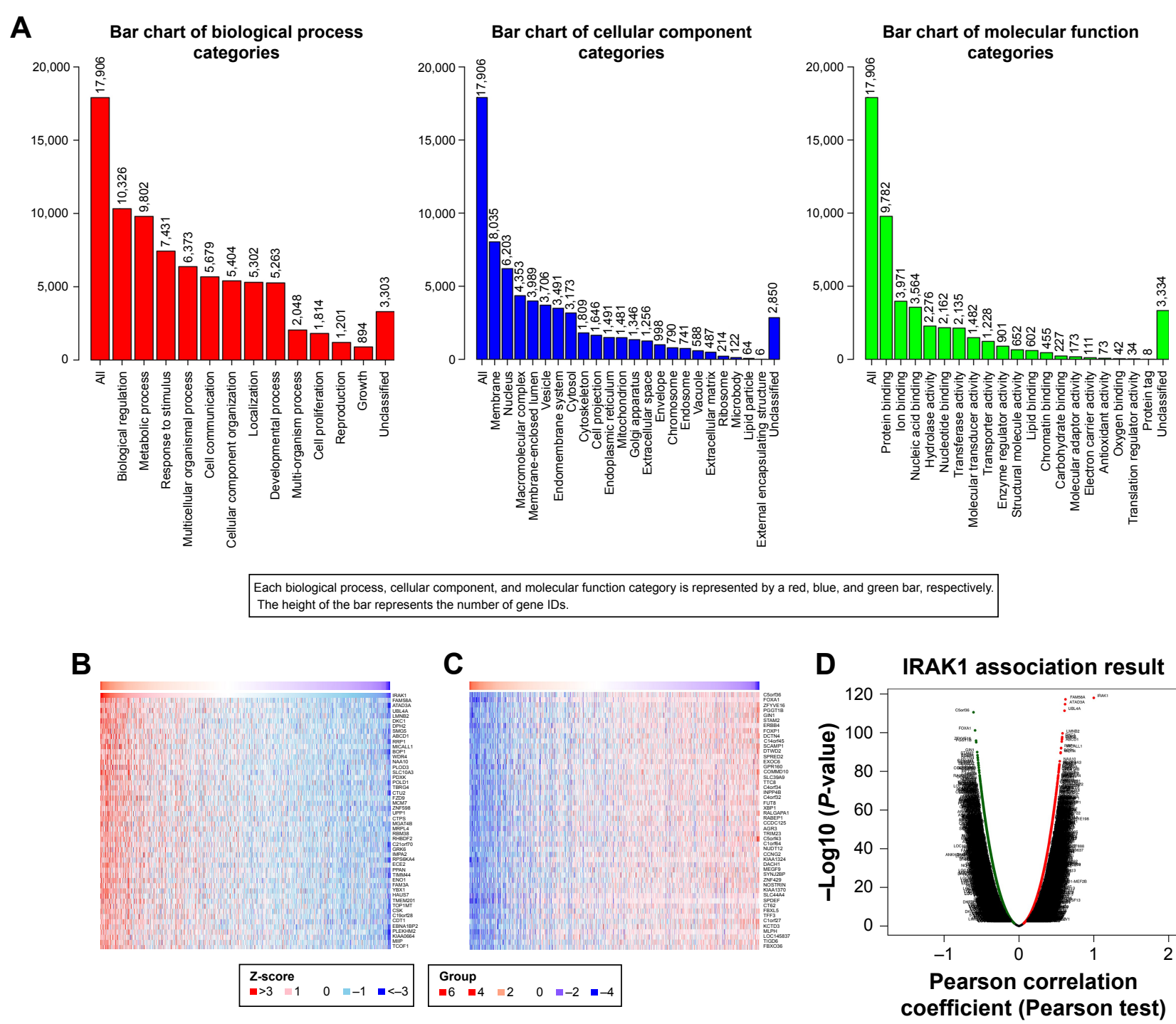

ach biological process, cellular component, and molecular function category is represented by a red, blue, and green bar, respectively. The height of the bar represents the number of gene IDs.

B
C
D IRAK1 association result

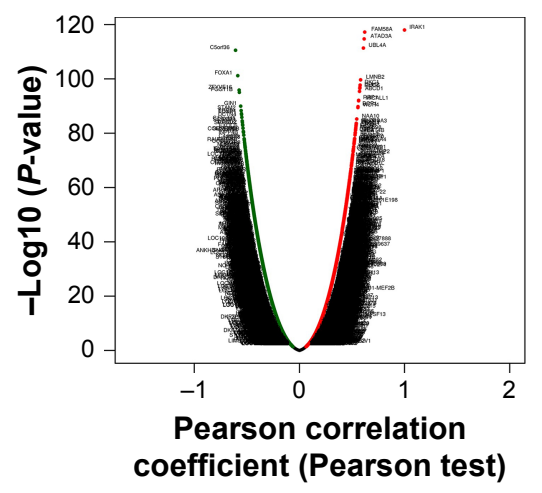

Figure 7 The enrichment results of IRAKI using web-based Gene SeT AnaLysis Toolkit.

Notes: (A) IRAKI enrichment in biological process, cellular component, and molecular function. (B) Positively related genes of IRAKI. (C) Negatively related genes of IRAKI. (D) Volcano plot of IRAKI in breast cancer.

cancer patients in TCGA database. The prognosis of IRAK1 in NCT patients was analyzed online with km-plotter. Immunohistochemical method was used to detect the expression pattern of IRAK1 in NCT breast cancer patients. The results showed that the expression of IRAK1 after NCT was significantly lower than before NCT, and the decreased expression of IRAK1 was correlated with change of tumor size. IRAK1 may be a potential prognostic indicator of the efficacy of NCT. The Human Protein Atlas validated IRAK1 protein expression in breast cancer tissue. The IRAK1 mutations were analyzed online using cBioPortal database to understand IRAK1 mutations in breast cancer and their association with survival. The IRAK1 protein interaction network was obtained through the String database, and its upstream and downstream related proteins were predicted and verified in
TCGA database. Gene enrichment analysis of IRAK1 was performed using the web-based Gene SeT AnaLysis Toolkit to understand its biological process. These bioinformatics analyses partially revealed the mechanism of IRAK 1 in breast cancer. However, the molecular mechanism of IRAK1 in NCT for breast cancer remains unclear. Bioinformatics analysis showed that the mutation rate of IRAK1 gene in breast cancer was as high as 7\%, and most of the mutant forms were amplified. Significantly altered neighboring genes within the network are APK2, MAP2K6, MAP2K4, MAP3K3, AKT3, MTOR, PRKCA, and TLR5. Therefore, IRAK1 may also promote the development of breast cancer through the MAP3K-MAP2K-MAPK signaling pathway and the AKTmTOR signaling pathway in addition to the TLR signaling pathway. Enrichment analysis suggested that IRAK1 plays a 
major role in biological regulation, metabolic processes, and protein binding. These findings will guide our further study in exploring the mechanism of action of IRAK1 in NCT.

\section{Disclosure}

We declare that we have no financial and personal relationships with other people or organizations that can inappropriately influence our work. There is no professional or other personal interest of any nature or kind in any product, service, and/or company that could be construed as influencing the position presented in, or the review of the manuscript. The authors report no other conflicts of interest in this work.

\section{References}

1. Chen W, Zheng R, Baade PD, et al. Cancer statistics in China, 2015. CA Cancer J Clin. 2016;66(2):115-132.

2. Bartsch R, Ziebermayr R, Zielinski CC, Steger GG. Triple-negative breast cancer. Wien Med Wochenschr. 2010;160(7-8):174-181.

3. Zardavas D, Piccart M. Neoadjuvant therapy for breast cancer. Annu Rev Med. 2015;66:31-48.

4. Mamounas EP. Impact of neoadjuvant chemotherapy on locoregional surgical treatment of breast cancer. Ann Surg Oncol. 2015;22(5): 1425-1433.

5. Rapoport BL, Demetriou GS, Moodley SD, Benn CA. When and how do I use neoadjuvant chemotherapy for breast cancer? Curr Treat Options Oncol. 2014;15(1):86-98.

6. Redden MH, Fuhrman GM. Neoadjuvant chemotherapy in the treatment of breast cancer. Surg Clin N Am. 2013;93(2):493-499.

7. Kaufmann M, von Minckwitz G, Mamounas EP, et al. Recommendations from an international consensus Conference on the current status and future of neoadjuvant systemic therapy in primary breast cancer. Ann Surg Oncol. 2012;19(5):1508-1516.

8. Janssens S, Beyaert R. Functional diversity and regulation of different interleukin-1 receptor-associated kinase (IRAK) family members. Molecular Cell. 2003;11(2):293-302.

9. Zhu J, Mohan C. Toll-like receptor signaling pathways-therapeutic opportunities. Mediators Inflamm. 2010;2010:781235.

10. Srivastava R, Geng D, Liu Y, et al. Augmentation of therapeutic responses in melanoma by inhibition of IRAK-1, -4. Cancer Res. 2012; 72(23):6209-6216.

11. Cheng BY, Lau EY, Leung HW, et al. IRAK1 augments cancer stemness and drug resistance via the AP-1/AKR1B10 signaling cascade in hepatocellular carcinoma. Cancer Res. 2018;78(9):2332-2342.

12. Chou CK, Chi SY, Huang CH, et al. IRAK1, a target of miR-146b, reduces cell aggressiveness of human papillary thyroid carcinoma. J Clin Endocrinol Metab. 2016;101(11):4357-4366.

13. Zhang X, Dang Y, Li P, Rong M, Chen G. Expression of IRAK1 in lung cancer tissues and its clinicopathological significance: a microarray study. Int J Clin Exp Pathol. 2014;7(11):8096-8104.

OncoTargets and Therapy

\section{Publish your work in this journal}

OncoTargets and Therapy is an international, peer-reviewed, open access journal focusing on the pathological basis of all cancers, potential targets for therapy and treatment protocols employed to improve the management of cancer patients. The journal also focuses on the impact of management programs and new therapeutic agents and protocols on

Submit your manuscript here: http://www.dovepress.com/oncotargets-and-therapy-journal
14. Pilarsky C, Wenzig M, Specht T, Saeger HD, Grützmann R. Identification and validation of commonly overexpressed genes in solid tumors by comparison of microarray data. Neoplasia. 2004;6(6):744-750.

15. Wee ZN, Yatim SM, Kohlbauer VK, et al. IRAK1 is a therapeutic target that drives breast cancer metastasis and resistance to paclitaxel. Nat Commun. 2015;6(1):8746.

16. Nenutil R. [A revolution postponed indefinitely. WHO classification of tumors of the breast 2012: the main changes compared to the 3rd edition (2003)]. Cesk Patol. 2015;51(1):23-25.

17. Yu NY, Hallström BM, Fagerberg L, et al; Fantom Consortium. Complementing tissue characterization by integrating transcriptome profiling from the human protein Atlas and from the FANTOM5 Consortium. Nucleic Acids Res. 2015;43(14):6787-6798.

18. Cerami E, Gao J, Dogrusoz U, et al. The cBio cancer genomics portal: an open platform for exploring multidimensional cancer genomics data. Cancer Discov. 2012;2(5):401-404.

19. Sirintrapun SJ, Zehir A, Syed A, Gao J, Schultz N, Cheng DT. Translational bioinformatics and clinical research (biomedical) informatics. Clin Lab Med. 2016;36(1):153-181.

20. Belizário JE, Sangiuliano BA, Perez-Sosa M, Neyra JM, Moreira DF. Using pharmacogenomic databases for discovering Patient-Target genes and small molecule candidates to cancer therapy. Front Pharmacol. 2016;7:312.

21. Szklarczyk D, Franceschini A, Kuhn M, et al. The string database in 2011: functional interaction networks of proteins, globally integrated and scored. Nucleic Acids Res. 2011;39(Database issue):D561-D568.

22. Szklarczyk D, Franceschini A, Wyder S, et al. String v10: protein-protein interaction networks, integrated over the tree of life. Nucleic Acids Res. 2015;43(Database issue):D447-D452.

23. Tang Z, Li C, Kang B, Gao G, Li C, Zhang Z. GEPIA: a web server for cancer and normal gene expression profiling and interactive analyses. Nucleic Acids Res. 2017;45(W1):W98-W102.

24. Zhang B, Kirov S, Snoddy J. WebGestalt: an integrated system for exploring gene sets in various biological contexts. Nucleic Acids Research. 2005;33(Web Server issue):W741-W748.

25. Wang J, Duncan D, Shi Z, Zhang B. Web-based gene set analysis toolkit (WebGestalt): update 2013. Nucleic Acids Res. 2013;41(Web server issue):W77-W83.

26. West AC, Tang K, Tye H, et al. Identification of a TLR2-regulated gene signature associated with tumor cell growth in gastric cancer. Oncogene. 2017;36(36):5134-5144.

27. Scheeren FA, Kuo AH, van Weele LJ, et al. A cell-intrinsic role for TLR2-MYD88 in intestinal and breast epithelia and oncogenesis. Nat Cell Biol. 2014;16(12):1238-1248.

28. Rajput S, Volk-Draper LD, Ran S. TLR4 is a novel determinant of the response to paclitaxel in breast cancer. Mol Cancer Ther. 2013;12(8): 1676-1687.

29. Rhyasen GW, Bolanos L, Fang J, et al. Targeting IRAK1 as a therapeutic approach for myelodysplastic syndrome. Cancer Cell. 2013;24(1): 90-104.

30. Ye ZH, Gao L, Wen DY, et al. Diagnostic and prognostic roles of IRAK1 in hepatocellular carcinoma tissues: an analysis of immunohistochemistry and RNA-sequencing data from the cancer genome atlas. Onco Targets Ther. 2017;10:1711-1723.

patient perspectives such as quality of life, adherence and satisfaction The manuscript management system is completely online and includes a very quick and fair peer-review system, which is all easy to use. Visit http://www.dovepress.com/testimonials.php to read real quotes from published authors. 